\title{
First-Order Transitions in Spherical Models: Finite-Size Scaling
}

\author{
Michael E. Fisher ${ }^{1 \dagger}$ and Vladimir Privman ${ }^{2 \star}$ \\ 1 Department of Theoretical Physics, University of Oxford, 1 Keble Road, Oxford OX1 3NP, England \\ 2 Downs Laboratory of Physics 405-47, California Institute of Technology, Pasadena, California 91125, \\ USA
}

\begin{abstract}
Finite-size behavior near the first-order phase boundary of ferromagnetic spherical models is investigated for block- and cylinder-shaped systems in $d$ dimensions. The bulk thermodynamic singularities are rounded and, asymptotically for large size, obey appropriate scaling laws. Both shortrange interactions and long-range couplings, decaying like $1 / r^{d+\sigma}$ with $\sigma>0$, are analyzed: the short-range results agree precisely with a recently developed scaling theory for $O(n)$ symmetric systems in the limit $n \rightarrow \infty$. More generally, the scaling functions are universal, depending only on $\sigma$. Explicit aspects of the shape and interactions enter only in the "spin wave" or "Goldstone mode" contributions which appear, technically, as "corrections to scaling." An appendix analyzes the truncation error in the approximation, by many-fold sums, of multivariate integrals with integrands diverging like $\left[\sum_{j} a_{j} \theta_{j}^{2}\right]^{-\lambda}$ as $\boldsymbol{\theta} \rightarrow 0$.
\end{abstract}

\section{Introduction}

The spherical model of a ferromagnet [1] was devised by Kac and is of interest, in particular, because it can be solved in closed form in the thermodynamic limit [2-5]. For dimensionality, $d$, exceeding $d=2$, it exhibits a phase transition if the interactions are of short range. However, it is soluble also for long-range, power-law interactions. Furthermore, it exhibits a nontrivial variation of the critical exponents with dimensionality and interaction decay, which makes it a valuable tool in studying bulk and surface critical behavior $[6,7]$. The model is also tractable when one or more of the linear dimensions are large but finite. Thus it has been employed to test the theory of finite-size scaling $[8,9]$ which describes the rounding or distortion of the bulk critical singularities that must occur in a system with restricted dimensions. These developments have been reviewed by Barber [10]; see also [11-13] for some more recent studies.

† Permanent address: Baker Laboratory, Cornell University, Ithaca, New York 14853, USA

* Present address: Physics Department, Clarkson University, Potsdam, New York 13676, USA 
Another well known property of the spherical model is its equivalence to the properly normalized limit of the $O(n)$-symmetric vector spin model when $n \rightarrow \infty$ $[14,15]$. This correspondence is exact in the thermodynamic limit [15], but is also valid for systems of finite size provided all spins are equivalent under some symmetry operation. For the standard space lattices this is ensured by periodic boundary conditions. It is of especial interest to us here that a bulk ferromagnetic spherical model exhibits singularities, such as a diverging susceptibility when the first-order transition boundary is approached by letting the magnetic field, $H$, vanish at temperatures, $T(>0)$, below the bulk critical temperature, $T_{c}$. These singularities are the direct analog of the so-called "spin-wave" or "Goldstone mode" singularities which characterize all systems with broken $O(n)$ symmetry for $n \geqq 2$.

The present work studies the rounding of the first-order transition and of the "spin-wave" singularities in ferromagnetic, $d$-dimensional spherical models with large but finite dimensions. We focus on those aspects that can be used to check the predictions of a general finite-size scaling theory $[16,17]$ for $O(n)$ symmetric models in the vicinity of a bulk first-order transition that breaks the symmetry. This theory extended a similar analysis [18] for $(n=1)$-component or scalar spin systems. (For further references, see the literature cited in [16-18].) For a "block" system of volume $V=L_{0}^{d}$ that has all linear dimensions $L_{1}, L_{2}, \ldots, L_{d}$ comparable as $V \rightarrow \infty$, the theory predicts that the singular part of the reduced free energy density should obey the asymptotic scaling law

$$
f_{s}(T, H) \approx V^{-1} W_{(n)}\left(y_{V}\right),
$$

in which the basic scaling combination is given by

$$
y_{V}=m_{0}(T) H V / k_{B} T,
$$

while $m_{0}(T)$ is the spontaneous magnetization density (and $k_{B}$ is Boltzmann's constant). The scaling function $W_{(n)}(\cdot)$ is expected to depend on $n$ but not on other details of the system. However, in the case of a "cylinder" geometry in which one dimension, $L_{\|} \equiv L_{1}$, greatly exceeds the others, a second scaling combination should be needed in (1.1), namely,

$$
x=L_{\|} / \xi_{\|}(T ; A) .
$$

For $n \geqq 2$, the special correlation length entering here is given by

$$
\xi_{\|}(T ; A)=2 \Upsilon_{(n)}(T) A /(n-1) k_{B} T,
$$

where $\Upsilon_{(n)}(T)$ is the helicity modulus [19] or "spin wave stiffness," while $A=$ $L_{2} L_{3} \cdots L_{d}$ is the cross-sectional area of the cylinder. We will, in fact, prove that these expectations are fully valid for the spherical model. In addition, we will obtain explicit expressions for the scaling functions, including the extensions that account for the rounding of the spin wave singularities: these are missing in (1.1) since they occur on a scale of magnetic field set by the new combination $y_{S}=$ $m_{0}(T)|H| L_{0}^{2} / \Upsilon_{(n)}(T)$ being of the order unity. Both short-range interactions, as contemplated in the general theory, and long-range power law interactions decaying like $1 / r^{d+\sigma}$ with $\sigma>0$ will be analyzed.

In outline we proceed as follows: known results for the thermodynamics of finite 
spherical models on hypercubical lattices $[5,6]$ and various definitions of range and shape parameters are presented in Sect. 2. Section 3 is devoted to analyzing the rounding of the first-order transition in block shapes. The leading scaling behavior is easily found, but the nature and rounding of the spin-wave singularities requires further work. The analysis rests on the Appendix which obtains expressions for the discretization errors in the approach of various many-fold sums to limiting multidimensional integrals with diverging integrands: these discretization errors enter directly in the calculations of the finite-size scaling functions describing the rounded spin-wave regime, in the corrections in the first-order regime, and in the full scaling-with-corrections form in terms of $y_{V}$ and $u=\left|y_{S} / y_{V}\right|$ (applicable when $d<$ $2 \sigma$ ). The rounding of the first-order transition in a long cylinder is taken up in Sect. 4. Explicit scaling expressions for the limiting behavior and for the crossover to block shape are obtained. In contrast to the block limit, the scaling function describing the rounding of the transition in leading order depends on the interaction exponent $\sigma$.

\section{Equation of State for Finite Spherical Models}

This section describes the models of interest and summarizes the results and definitions needed for the subsequent calculations. We address the thermodynamics of spherical models defined on $d$-dimensional simple hypercubical lattices of spacing $a$ and finite dimensions, denoted $\mathbf{L}$ or $\mathbf{N}$, on which periodic boundary conditions are imposed. Specifically, we write

$$
\begin{aligned}
& \mathbf{L}=\left\{L_{k}\right\}_{k=1, \ldots, d}, \quad \mathbf{N}=\left\{N_{k}\right\}_{k=1, \ldots, d}, \quad L_{k}=N_{k} a, \\
& V=\prod_{k=1}^{d} L_{k} \equiv L_{0}^{d}, \quad N=\prod_{k=1}^{d} N_{k} \equiv N_{0}^{d}, \quad V=N a^{d} .
\end{aligned}
$$

Each lattice site $i$, with cartesian coordinate vector $\mathbf{r}_{(i)}=\left(r_{(i) k}\right)(k=1, \ldots, d)$, has an associated continuous scalar "spin" variable $s_{i}$. The spins interact via the Hamiltonian

$$
\mathscr{H}=-\sum_{(i, j)} J\left(\mathbf{r}_{(j)}-\mathbf{r}_{(i)}\right) s_{i} s_{j}-H a^{d} \sum_{i=1}^{N} s_{i}-\zeta \sum_{i=1}^{N} s_{i}^{2},
$$

in which the sum on $(i, j)$ runs over all distinct pairs of sites. The factor $a^{d}$ is included for convenience in comparing with the general scaling theories [16-18]. The coefficient $\zeta$ represents the so-called spherical field. At all $T$ and $H$ it is fixed by imposing the mean spherical constraint [4-6]

$$
\sum_{i}\left\langle s_{i}^{2}\right\rangle=V / a^{d} \equiv\left(L_{0} / a\right)^{d},
$$

in which $\langle\cdot\rangle$ denotes a thermal average computed with $\mathscr{H}$.

The Interactions. The interactions, $J(\mathbf{r})$, enter the exact expressions for the free energy only through the Fourier transform

$$
\hat{J}(\mathbf{q})=\sum_{i} e^{i \mathbf{q} \cdot \mathbf{r}_{(i)}} J\left(\mathbf{r}_{(i)}\right)
$$


In order to discuss the approach to the thermodynamic limit, $\mathbf{L} \rightarrow \infty$, we thus choose to define the interactions by specifying the transform $\hat{J}(\mathbf{q})$, over the Brillouin zone, which we fix, independent of $\mathbf{L}$. The original couplings, $J(\mathbf{r})$, appearing in $(2.3)$ then follow by inverting (2.5) by summing, for a finite periodic system, over the appropriate set of discrete wave vectors [see (2.14)-(2.16) below]. Unless, in the infinite system, $J(\mathbf{r})$ is of strictly finite range (i.e. of bounded support) this means that $J(\mathbf{r})$ in a finite system contains a definite but asymptotically negligible dependence on $\mathbf{L}$ corresponding, physically, to summing over interactions with all periodically repeated images. To describe "simple ferromagnetic interactions" we assume that $\hat{J}(\mathbf{q})$ attains a unique maximum, $\hat{J}(0)$, at $\mathbf{q}=0$ and is analytic for $\mathbf{q} \neq 0$. To characterize the long-distance decay of the potentials, which affects the existence of a transition and the values of the exponents describing the spin wave and critical singularities, we introduce a positive exponent $\sigma$ and assume

$$
\widehat{J}(\mathbf{q})=\widehat{J}(0)\left[1-(2 d)^{-1}\left(\sum_{k=1}^{d} R_{k}^{2} q_{k}^{2}\right)^{\sigma / 2}+O\left(|\mathbf{q}|^{\sigma+\delta}\right)\right],
$$

with $\delta>0$. The value $\sigma=2$ (with, typically, $\delta=2$ ) characterizes short-range interactions; it follows that values $\sigma>2$ are nonphysical in general, although the analysis is not affected. Values satisfying $0<\sigma<2$ correspond to long-range potentials decaying essentially like $1 / r_{0}{ }^{d+\sigma}$ with

$$
r_{0}^{2}=\left[\left(r_{1} / R_{1}\right)^{2}+\left(r_{2} / R_{2}\right)^{2}+\cdots+\left(r_{d} / R_{d}\right)^{2}\right] .
$$

Evidently the $R_{k}$ specify the anisotropy of the interactions. It transpires that there is no phase transition unless

$$
\sigma<d,
$$

which condition we shall thus presuppose whenever needed.

The Equation of State. The computation of the partition function, $Z$, for the spherical model as specified here is described in detail in Refs. [4-6]. From the reduced free energy density, $f=F / k_{B} T V=-V^{-1} \ln Z$, one computes the magnetization per spin

$$
m(H, T ; \mathbf{L})=N^{-1} \sum_{i}\left\langle s_{i}\right\rangle=-(\partial f / \partial h)_{T},
$$

where it proves convenient to put

$$
h=H / k_{B} T \text { and } K=\widehat{J}(0) / 2 d k_{B} T .
$$

The result can be written

$$
m=a^{d} H d / \widehat{J}(0) \phi=a^{d} h / 2 K \phi,
$$

where $\phi(H, T ; \mathbf{L})$ represents the appropriately shifted and scaled spherical field [6], which must be determined via the constraint

$$
2 K\left(1-m^{2}\right)=\frac{a^{d}}{V} \sum_{\mathbf{q}} \frac{1}{\phi+\Omega_{\mathrm{q}}},
$$


in which the interactions enter through

$$
\boldsymbol{\Omega}_{\mathbf{q}} \equiv \boldsymbol{\Omega}(\boldsymbol{\theta})=2 d[1-\hat{J}(\mathbf{q}) / \hat{J}(0)]
$$

The wavevector sum runs over the discrete values specified by

$$
\mathbf{q} a \equiv \boldsymbol{\theta} \equiv \boldsymbol{\theta}(\mathbf{p})=\left(\frac{2 \pi}{N_{1}} p_{1}, \ldots, \frac{2 \pi}{N_{d}} p_{d}\right),
$$

where the integer vector, $\mathbf{p} \equiv\left(p_{k}\right)$, is determined by

$$
p_{k}=0, \pm 1, \pm 2, \ldots, \quad\left(\bmod N_{k}\right) \text {. }
$$

Thus one has the alternative form

$$
\left(a^{d} / V\right) \sum_{q} \equiv N_{0}^{-d} \sum_{p_{1}=0}^{N_{1}-1} \cdots \sum_{p_{d}=0}^{N_{d}-1} \equiv N^{-1} \sum_{\mathbf{p}} .
$$

It is convenient to specify dimensionless interaction ranges, $\rho_{k}$, via

$$
R_{0}^{d} \equiv \prod_{k=1}^{d} R_{k} \quad \text { and } \quad \rho_{k}=R_{k} / a, \quad(k=0,1, \ldots, d)
$$

and system shape ratios, $\mathbf{I}=\left\{l_{k}\right\}$, by

$$
l_{k}=L_{k} / L_{0}=L_{k} / V^{1 / d}=N_{k} / N_{0}
$$

Then as $|\boldsymbol{\theta}| \rightarrow 0$, one has, from (2.6),

$$
\Omega(\boldsymbol{\theta}) \approx\left[\sum_{k=1}^{d} \rho_{k}^{2} \theta_{k}^{2}\right]^{\sigma / 2}
$$

where here, as usually below, we do not display explicitly the error estimates following from the $|\mathbf{q}|^{\sigma+\delta}$ term in (2.6). For small $|\mathbf{p}| / N_{0}$ this may be transcribed into the useful form

$$
\Omega(\boldsymbol{\theta}) \approx\left(\frac{2 \pi \rho_{0}}{N_{0}}\right)^{\sigma}\left[\sum_{k=1}^{d} \omega_{k} p_{k}^{2}\right]^{\sigma / 2},
$$

which serves to show that the finite-size effects depend, asymptotically, only on the parameters $\omega=\left\{\omega_{k}\right\}$ with

$$
\omega_{k}=\left(\rho_{k} / \rho_{0} l_{k}\right)^{2}=\left(R_{k} / L_{k}\right)^{2} /\left(R_{0} / L_{0}\right)^{2} .
$$

For interactions and system shapes respecting full cubic symmetry one has $\omega_{k} \equiv 1$ (all $k$ ).

Finally, note that in the thermodynamic limit, $\mathbf{L} \rightarrow \infty$, taken with $\phi \neq 0$, the sum in (2.12) becomes an integral over the basic zone. Eliminating $\phi$ with (2.11) then yields an implicit equation for the magnetization $m(H, T)$. As $H \rightarrow 0+$ below $T_{c}, m$ should approach the spontaneous magnetization $m_{0}(T)$ : if this is nonvanishing we obtain from (2.11),

$$
\phi \approx a^{d} h / 2 K m_{0}(T), \text { as } H \rightarrow 0+
$$


for $T<T_{c}$. From (2.12) the spontaneous magnetization must then be given by

$$
m_{0}^{2}(T)=1-\frac{d k_{B} T}{\widehat{J}(0)} \int_{\Theta} \Omega^{-1}(\boldsymbol{\theta})
$$

where $\int_{\Theta}=\int d^{d} \theta /(2 \pi)^{d}$ denotes a multiple integral over the rescaled zone $\Theta=$ $\left\{\boldsymbol{\theta} ;\left|\theta_{k}\right|<\pi\right\}$. The critical temperature, at which $m_{0}(T) \rightarrow 0+$ follows from

$$
2 K_{c} \equiv \widehat{J}(0) / d k_{B} T_{c}=\int_{\Theta} \Omega^{-1}(\theta) .
$$

The integral here is formally divergent for $d \leqq \sigma$ which, on paying closer attention to the details, shows (as mentioned) that there is no transition unless $\sigma<d$. On combining (2.22) and (2.23) one finds simply

$$
m_{0}(T)=\left[1-\left(T / T_{c}\right)\right]^{1 / 2} .
$$

\section{Rounding of the First-Order Transition in Block Geometries}

We will now investigate the form of the rounding of the first-order transition below $T_{c}$ that occurs in block geometries: more precisely we consider the limit $V, L_{0} \rightarrow \infty$ with the shape ratios, $l_{k}=L_{k} / L_{0}$, asymptotically fixed and nonvanishing.

Now, as seen in deriving (2.23), the vicinity of the phase boundary corresponds to $\phi$ small and $T<T_{c}$. As $\phi \rightarrow 0$ in a finite system the $\mathbf{q}=0$ term in the sum in (2.12) diverges. Accordingly, in the equation of state (2.12) we separate off this term, $a^{d} / V \phi=1 / N \phi$, and subtract expression (2.23) for $m_{0}^{2}(T)$. Gathering up matching terms then yields

$$
2 K\left(m_{0}^{2}-m^{2}\right)=1 / N \phi-e_{0}(\mathbf{N})-\phi U_{1}(\phi ; \mathbf{N}),
$$

in which all the dependences on $T$, or $K, \phi$ and $\mathbf{L}=\mathbf{N} a$ have been displayed while, more generally,

$$
e_{i}(\mathbf{N})=\int_{\Theta} \frac{1}{[\Omega(\boldsymbol{\theta})]^{i+1}}-\frac{1}{N} \sum_{p \neq 0} \frac{1}{[\Omega(\boldsymbol{\theta})]^{i+1}},
$$

provided $(i+1) \sigma<d$ so the integral exists, and

$$
U_{i}(\phi ; \mathbf{N})=\frac{1}{N} \sum_{\mathbf{p} \neq 0} \frac{1}{[\phi+\Omega(\boldsymbol{\theta})][\Omega(\boldsymbol{\theta})]^{i}} .
$$

Our main task is thus to analyze $e_{0}$, the difference between an integral with a divergent integrand and an approximating sum, and the sum $U_{1}(\phi)$ which, since $d>\sigma$, should be well approximated by an integral as $\mathbf{N} \rightarrow \infty$ provided $\phi>0$. The former problem is considered generally in the Appendix from which one finds that $e_{0}$ vanishes as $1 / N_{0}^{d-\sigma}$ : see further below. The latter question is taken up in detail shortly: here we observe merely that when $\phi \rightarrow 0$ one has $\phi U_{1}(\phi)=O\left(\phi^{(d-\sigma) / \sigma}\right) \rightarrow 0$.

Leading Scaling Behavior. The observations regarding $e_{0}$ and $U_{1}$ enable us to obtain the leading behavior near the first order transition directly. For finite $\mathbf{N}$ and 
$\phi \rightarrow 0$ the first term on the right of (3.1) dominates $e_{0}$ and $\phi U_{1}$. Then by using (2.11) we obtain the asymptotic equation of state,

$$
m_{0}^{2}-m^{2} \approx m / h V
$$

where the nature of the corrections will be made precise later. This result may be cast in the anticipated scaling form

$$
m(H, T ; \mathbf{L}) \approx m_{0}(T) Y_{0}\left(y_{V}\right),
$$

where the appropriate scaling combination of magnetic field and volume is

$$
y_{V}=m_{0}(T) V H / k_{B} T \equiv H / H_{V},
$$

just as expected generally [16-18]. The scaling function is found to be

$$
Y_{0}(y)=2 y /\left[1+\left(1+4 y^{2}\right)^{1 / 2}\right]
$$

which also agrees precisely with the prediction of the phenomenological theory [16].

To interpret these results [16-18] note the limiting forms.

$$
\begin{aligned}
Y_{0}(y) & =y+O\left(y^{3}\right), & & \text { as } y \rightarrow 0, \\
& = \pm\left[1-\frac{1}{2}|y|^{-1}+O\left(|y|^{-2}\right)\right], & & \text { as } y \rightarrow \pm \infty .
\end{aligned}
$$

It follows from these, with (3.6) and (3.5), that for $|H| \gg H_{V} \sim 1 / V$ the magnetization takes the values $\operatorname{sgn}(H) m_{0}(T)$, while for $H \ll H_{V}$ it passes smoothly through 0 ; note that the zero-field susceptibility diverges as

$$
\chi(H=0, T ; \mathbf{L}) \approx m_{0}^{2}(T) V / k_{B} T,
$$

when $V \rightarrow \infty$. Thus the jump from $-m_{0}$ to $+m_{0}$ across the phase boundary in the limiting bulk magnetization is smoothly rounded off, as it must be in a finite system, on a scale $|H| \simeq H_{V}$. Note, further, that none of the leading asymptotic behavior at the first-order transition depends on the dimensionality, on the actual shape of the lattice, or on the details of the interaction decay or anisotropy.

Spin Wave Singularities. Before studying the corrections to the leading scaling behavior it is appropriate to examine the full equation of state, (2.12), in the thermodynamic limit and to obtain the behavior of the magnetization for small $H$ (below $T_{c}$ ). This entails the integral

$$
Q^{\infty}(\phi) \equiv \int_{\Theta} \frac{1}{[\phi+\Omega(\theta)]}=2 K_{c}-\phi U_{1}(\phi ; \infty),
$$

which is of a well-studied type [e.g.: 5, 6, 20]. The first $I-1$ derivatives of $Q^{\infty}(\phi)$ are bounded at $\phi=0+$, where $I \equiv I(d, \sigma)$ is the integer defined by

$$
I \sigma<d \leqq(I+1) \sigma .
$$

For $d \neq(I+1) \sigma$, one readily finds

$$
Q^{\infty}(\phi)=b_{0}-b_{1} \phi+\cdots+b_{I-1}(-\phi)^{I-1}+b_{*} \phi^{(d-\sigma) / \sigma}[1+o(1)]
$$

as $\phi \rightarrow 0$; on the borderline $d=(I+1) \sigma$ the final, singular term becomes $b_{*} \phi^{I}(\ln \phi) \times$ $[1+o(1)]$. The coefficients $b_{0} \equiv 2 K_{c}, b_{1}, \ldots, b_{I-1}$ depend on the details of $\hat{J}(\mathbf{q})$; 
however, the singular amplitude $b_{*}$ is a function only of $d, \sigma$, and the range ratio $\rho_{0}$ : see (3.22) et seq.

By (2.22) $\phi$ varies as $h / m_{0}$ when $h \rightarrow 0+$ in the thermodynamic limit, so we conclude that the bulk isotherm has a parallel expansion of the form

$$
\begin{aligned}
m(T, H)= & m_{0}(T)+\chi_{1}(T) H+\frac{1}{2} \chi_{2}(T) H^{2}+\cdots+\frac{1}{(I-1) !} \chi_{I-1}(T) H^{I-1} \\
& +\frac{1}{I !} \chi_{*}(T) H^{(d-\sigma) / \sigma}[1+o(1)],
\end{aligned}
$$

for $H \rightarrow 0+$. Note, in particular, that the $I^{\text {th }}$ order nonlinear susceptibility $\chi_{I}(T, H)=$ $\left(\partial^{I} \mathrm{~m} / \partial H^{I}\right)_{T}$ diverges as the phase boundary is approached. This divergence is a symptom of the 'spin wave' singularities characterizing broken $O(n)$ symmetric systems for all $n \geqq 2$ including $n \rightarrow \infty[16,19]$. These singularities must, clearly, also be rounded in a finite system, but the leading scaling form (3.5)-(3.7) contains no hint of them. We will show, in fact, that they are rounded but primarily on a second scale, $H_{S}$, which is asymptotically much larger than $H_{V}$ (although both vanish as $V \rightarrow \infty$ ).

Asymptotics of $e_{i}$ and $U_{i}$. To proceed further one needs more concrete information about $e_{0}(\mathbf{N})$ and $U_{1}(\phi ; \mathbf{N})$ : these functions may be conveniently regrouped to mirror (3.10) as

$$
Q(\phi ; \mathbf{N})=2 K_{c}-e_{0}(\mathbf{N})-\phi U_{1}(\phi ; \mathbf{N})=\frac{1}{N} \sum_{\mathbf{p} \neq 0} \frac{1}{\phi+\Omega(\boldsymbol{\theta})} .
$$

One may then expand, in parallel to (3.12), to obtain

$$
Q(\phi ; \mathbf{N})=\sum_{i=0}^{I-1}\left[b_{i}-e_{i}(\mathbf{N})\right](-\phi)^{i}+(-\phi)^{I} U_{I}(\phi ; \mathbf{N}),
$$

where $e_{i}(\mathbf{N})$ and $U_{I}(\phi, \mathbf{N})$ are given by (3.2) and (3.3).

As mentioned, the truncation error, $e_{i}(\mathbf{N})$, is analyzed for $N \equiv N_{0}^{d} \rightarrow \infty$ in the Appendix. One finds

$$
e_{i}(\mathbf{N}) \approx D(d,(i+1) \sigma ; \boldsymbol{\omega}) /\left(2 \pi \rho_{0}\right)^{(i+1) \sigma} N_{0}^{d-(i+1) \sigma},
$$

for $i=0,1, \ldots, I-1$ where for fixed $d, \sigma, \omega$ and $i$, the residual corrections decay more rapidly with $N_{0}$. Note that the $e_{i}(\mathbf{N})$ themselves decrease most rapidly for small $i$. Explicit expressions for the amplitude $D$ are constructed in the Appendix: it depends only on $d$, on $\sigma$, and on the individual shape/anisotropy ratios $\omega_{k}$ defined in (2.21).

Now the spin wave singularity in (3.12) arises from $U_{I}(\phi ; \mathbf{N})$ in the limit $\mathbf{N} \rightarrow \infty$, as a divergence of the limiting integral like $1 / \phi^{\lambda / \sigma}$ for $\lambda \equiv(I+1) \sigma-d>0$, but as $\ln \phi$ for $\lambda=0$. Since this divergence arises from small values of $\boldsymbol{\theta}$, we may invoke (2.20), which we write as

$$
\Omega\left(\boldsymbol{\theta}_{\mathrm{p}}\right) \approx\left[2 \pi \rho_{0} P_{\mathrm{p}}(\boldsymbol{\omega}) / N_{0}\right]^{\sigma} \quad \text { with } \quad P_{\mathrm{p}}^{2}(\boldsymbol{\omega})=\sum_{k=1}^{d} \omega_{k} p_{k}^{2},
$$

in order to obtain the leading singular behavior of $U_{I}$ as $\mathbf{N} \rightarrow \infty$. It is appropriate to 
define the scaled spherical field as

$$
v=\phi\left(N_{0} / \rho_{0}\right)^{\sigma}=\phi L_{0}^{\sigma} / R_{0}^{\sigma} .
$$

Then we expect $U_{I}(\phi ; \mathbf{N})$ to be approximated asymptotically by

$$
\tilde{U}_{I}(v)=\left(\frac{N_{0}}{\rho_{0}}\right)^{I \sigma} \frac{1}{\rho_{0}^{\sigma} N_{0}^{d-\sigma}} S(v ; \omega),
$$

where we presuppose $\lambda \equiv(I+1) \sigma-d \neq 0$, so that

$$
S(v ; \omega)=\sum_{\mathrm{p} \neq 0} \frac{\left[2 \pi P_{\mathrm{p}}(\omega)\right]^{-I \sigma}}{v+\left[2 \pi P_{\mathrm{p}}(\omega)\right]^{\sigma}} .
$$

In fact it is straightforward although tedious to examine the difference $U_{I}(\phi ; \mathbf{N})-$ $\tilde{U}_{I}(v)$, and to show that at fixed $v$ it vanishes as $\mathbf{N} \rightarrow \infty$. In this calculation it is natural to restrict $\mathbf{p}$ in (3.20) to the bounded domain (2.15). However, as $|\mathbf{p}| \rightarrow \infty$ the summand in (3.20) decays as $1 /|\mathbf{p}|^{(I+1) \sigma}$ when $|\mathbf{p}| \rightarrow \infty$ which, since $\lambda>0$, means the leading behavior is preserved if the sum is extended to all nonzero integer vectors: this extension will be understood as the meaning of (3.20). When $v \rightarrow \infty$ an approximating integral suffices for estimation and one finds

$$
S(v ; \omega) \approx G_{d, \sigma} v^{(d / \sigma)-I-1}, \quad \text { as } v \rightarrow \infty,
$$

there being no limiting dependence on $\omega$ since

$$
G_{d, \sigma}=\int \frac{d^{d} \psi}{(2 \pi)^{d} \psi^{I \sigma}\left(1+\psi^{\sigma}\right)}=\frac{\pi^{d / 2}(2 \pi)^{1-d}}{\sigma \Gamma\left(\frac{1}{2} d\right) \sin [\pi(d-I \sigma) / \sigma]} .
$$

From this one obtains $\phi^{I} \tilde{U}_{I} \approx G_{d, \sigma} \rho_{0}^{-d} \phi^{(d-\sigma) / \sigma}$, which reconfirms (3.12) and provides the result $b_{*}=(-)^{I} G_{d, \sigma} / \rho_{0}^{d}$.

In the borderline case $\lambda=(I+1) \sigma-d=0$, the truncated sum in (3.20) cannot be extended and, in place of (3.19), one obtains

$$
U_{I}(\phi ; \mathbf{N}) \approx \widetilde{U}_{I}(v)=\rho_{0}^{-d}\left[C_{d} \ln N_{0}+S_{*}(v ; \omega)\right],
$$

where the coefficient $C_{d}$ depends only on $d$, while $S_{*}$ is given by a form generalizing (3.20) which we will not explore. Indeed, since our main purpose is to study the generic scaling behavior, we will focus below only on the cases $d / \sigma$ nonintegral $(\lambda \neq 0)$. One knows from many studies that logarithmic factors commonly appear at borderline dimensionalities; but this is a technical complication which poses no real theoretical mysteries! Nevertheless, we should admit that for short range forces, when $\sigma=2$, we will not be exhibiting further results for the theoretically fashionable case $d=4$.

Finally, on assembling the results in (3.13) we may write (for $d / \sigma$ nonintegral)

$$
\begin{aligned}
Q(\phi ; N)-2 K_{c} & \equiv-e_{0}(\mathbf{N})-\phi U_{1}(\phi ; \mathbf{N}) \\
& \approx \sum_{i=1}^{I-1} b_{i}(-\phi)^{i}-\left(2 / \rho_{0}^{\sigma} N_{0}^{d-\sigma}\right) Y_{S}(v ; \omega),
\end{aligned}
$$

where the basic "spin wave" scaling function with argument (3.18) is

$$
Y_{S}(v ; d, \sigma ; \omega)=\frac{1}{2} \sum_{i=0}^{I-1}(2 \pi)^{-(i+1) \sigma} D(d,(i+1) \sigma ; \omega)(-v)^{i}-\frac{1}{2}(-v)^{I} S(v ; d, \sigma ; \omega),
$$


where now, if $[x]$ is the integral part of $x$, we can take $I=[d / \sigma]$, while $S$ is defined in (3.20) and $D$ in (A7). Recall also that the coefficients $b_{i}$ depend on the details of $\hat{J}(\mathbf{q})$ through (3.10) and (3.12).

Asymptotic Equation of State. We can now study all the corrections to the leading equation of state (3.4) by combining (3.24) and (2.11) in (3.1) to obtain, finally, the basic formula

$$
m_{0}^{2}-m^{2} \approx \frac{m}{V h}+\frac{1}{2 K} \sum_{i=1}^{I-1} b_{i}(h / m)^{i}-\frac{a^{d}}{K R_{0}^{\sigma} L_{0}^{d-\sigma}} Y_{S}\left(\frac{a^{d}}{2 K}\left(\frac{L_{0}}{R_{0}}\right)^{\sigma} \frac{h}{m} ; \omega\right),
$$

in which $\bar{b}_{i}=\left(-a^{d} / 2 K\right)^{i} b_{i}$ (and $d / \sigma$ is nonintegral). This result gives the limiting behavior and leading asymptotic corrections for small $|H|\left(\lesssim a^{-d} \hat{J}(0) m_{0}\right)$ by whatever route $L_{0}$ (or $V$ ) diverges. To study its implications note first that at fixed $H>0$ the first term on the right vanishes when $V \rightarrow \infty$, while the last, spin-wave term contributes asymptotically only through $S(v)$ with $v \rightarrow \infty$. Thus with the aid of (3.21) one checks that the bulk result (3.13) is reproduced, the spin wave singularity arising entirely from $S(v)$ and its amplitude, $\chi_{*}$, following from (3.22).

To proceed further, we introduce two additional scaling combinations, $y_{S}$ and $u$, and a new field scale, $H_{S}$ [16]. Thus, recalling (3.6), we rewrite (3.18) as

$$
v=y_{S}\left|m_{0} / m\right|=u y_{V}\left(m_{0} / m\right)
$$

through which the spin-wave scale, $H_{S}$, is set by

$$
y_{S}=\frac{a^{d}|h| N_{0}^{\sigma}}{2 K m_{0} \rho_{0}^{\sigma}}=\frac{d a^{d}|H| L_{0}^{\sigma}}{\hat{J}(0) m_{0} R_{0}^{\sigma}} \equiv \frac{|H|}{H_{S}},
$$

while the ratio of the scales is

$$
u(T ; \mathbf{L})=\frac{y_{S}}{\left|y_{V}\right|}=\frac{H_{V}}{H_{S}}=\left(\frac{d a^{d} k_{B} T}{\widehat{J}(0) m_{0}^{2}(T) R_{0}^{\sigma}}\right) \frac{1}{L_{0}^{d-\sigma}} .
$$

Note that this parameter is essentially the prefactor of the $Y_{S}$ term in (3.26): it is independent of $H$ and vanishes as $\mathbf{L} \rightarrow \infty$. In renormalization group terms $u$ represents the scaled form of the leading irrelevant variable which controls the dominant corrections to scaling (see [16]). For systems with short range interactions it can be written [16], using (2.25), as

$$
u(\mathbf{L})=k_{B} T / \Upsilon_{0}(T) L_{0}^{d-2}, \quad(\sigma=2),
$$

in which $\Upsilon_{0}(T)$ is an isotropic (geometric) average helicity modulus [19] derived from the individual helicity moduli,

$$
\Upsilon_{k}(T)=\left(\hat{J}(0) R_{k}^{2} / d a^{d}\right)\left[1-\left(T / T_{c}\right)\right], \quad(k=1, \ldots, d),
$$

which measure the response to an imposed "twist" of the order parameter along the lattice axes. For isotropic nearest neighbor couplings this formula has been established by Barber and Fisher [6]; an alternative derivation of the general result follows from the analysis of the next section.

Spin Wave Regime. In the regime $H \gg H_{V} \sim 1 / V$, there will be no evidence of 
rounding of the first-order discontinuity in $m(H, T ; \mathbf{L})$ but the spin wave singularities will be rounded when $H \lesssim H_{S}$. To see this explicitly note that the term $m / V h$ in (3.26) may now be dropped, the equation may be solved iteratively, and then to sufficient accuracy one may put $m=m_{0}$ in (3.27), the argument of $Y_{S}$ in (3.26). This yields

$$
m(H, T ; \mathbf{L}) \approx \operatorname{sgn}(H)\left[m_{0}(T)+\sum_{i=1}^{I-1} \frac{1}{i !} \chi_{i}(T)|H|^{i}+m_{0}(T) u(T ; \mathbf{L}) Y_{S}\left(y_{S} ; \omega\right)\right],
$$

where the bulk nonlinear susceptibilities, $\chi_{i}$, were introduced in (3.13). The sum in which they appear represents what, in critical point studies, is often called the "bulk analytic background." The last term evidently represents the spin-wave singularity rounded on the scale $|H| \simeq H_{S}$ set by $y_{S}=O(1)$ : recall that $Y_{S}$ is defined explicitly through (3.25) and (3.20).

Full Scaling Form. If one has $\sigma<d<2 \sigma$, so that $I=1$, the surn in (3.26) is absent and the spin-wave singularities in the bulk limit dominate the background terms. In that case both spin-wave and first-order rounding can be encompassed in a single scaling formulation. Specifically, with (3.29) and (3.6) we may write

$$
m(H, T ; \mathbf{L}) \approx m_{0}(T) Y\left(y_{V}, u ; \boldsymbol{\omega}\right),
$$

where the scaling function $Y(y, u)$ satisfies the equation

$$
Y^{2}+Y / y=1+2 u Y_{S}(u y / Y ; \omega) \text {. }
$$

Now $u(\mathbf{L})$ is small in all regimes as $\mathbf{L} \rightarrow \infty$, and so we may solve this equation by expansion in $u$ provided we preserve the "irrelevant but dangerous" $[16,18,21]$ combination $u y$ (which varies as $y_{S}$ when $\mathbf{L} \rightarrow \infty$ ). By iteration this yields

$$
Y(y, u)=Y_{0}(y)+u Y_{1}(y, u ; \omega)+O\left(u^{2}\right),
$$

where $Y_{0}$ is given by (3.7) while

$$
Y_{1}(y, u ; \omega)=2 y\left(1+4 y^{2}\right)^{-1 / 2} Y_{S}\left\{\frac{1}{2} u\left[1+\left(1+4 y^{2}\right)^{1 / 2}\right] ; \omega\right\} .
$$

These scaling expressions, valid for $d<2 \sigma$, confirm the $n \rightarrow \infty$ limiting behavior predicted by the general theory for $\sigma=2$ [16].

First-Order Regime. For $d>2 \sigma$ (or $I \geqq 2$ ) the sum of powers of $h / m$ in (3.26) plays a role and the corresponding background terms now dominate the spin-wave singularities in sufficiently large fields. An iterative procedure may still be used to find explicit approximations for $Y \equiv m / m_{0}$ if one substitutes $h / m=y_{V} / m_{0}^{2} Y V$, but the formulae are more complex because of the interference between "corrections" and "background." Simpler results can, however, be obtained in the rounded first-orderregion: $y_{V}=O(1)$ or $|h| \lesssim C / m_{0} V$ with $C$ bounded. Indeed, the ratio $|h / m|$ then ranges only from $1 / k_{B} T \chi(0, T ; \mathbf{L}) \approx 1 / m_{0}^{2} V$ in zero field, using (3.9), to $\left|h / m_{0}\right| \lesssim C / m_{0}^{2} V$, and so is negligible when $V \rightarrow \infty$. Accordingly, the sum in (3.26) can be dropped leaving the spin-wave term, $Y_{S}(v ; \omega)$, whose argument satisfies

$$
v \approx u y_{V} / Y_{0}\left(y_{V}\right) \approx u\left[1+\left(1+4 y_{V}^{2}\right)^{1 / 2}\right]=O(u) \rightarrow 0,
$$

as $\mathbf{L} \rightarrow \infty$. Thus to leading order in $u$ we require only $Y_{S}(0 ; \omega)$ which is represented by the $i=0$ term in (3.25). 
In summary, the rounding of the first-order transition on the scale $|H|=O\left(H_{V}\right)$ is described correct to first order in $u$ by

$$
m(H, T ; \mathbf{L}) \approx m_{0}(T) y_{V}\left[\frac{2}{1+\left(1+4 y_{V}^{2}\right)^{1 / 2}}+\frac{D(d, \sigma ; \boldsymbol{\omega}) u}{(2 \pi)^{\sigma}\left(1+4 y_{V}^{2}\right)^{1 / 2}}\right],
$$

where, as before, $y_{V}=m_{0} H V / k_{B} T$ and $D$ is given in (A.7). Inspection of the arguments yielding this result reveals that it is valid for all $d / \sigma>1$, since only the $i=0$ term in (3.25) is entailed: this is equivalent to $e_{0}(\mathbf{N})$ in (3.24) for which (3.16) remains correct even when $d=(I+1) \sigma$. By differentiation we extend the result (3.9) for the zero-field susceptibility to

$$
\chi(0, T ; \mathbf{L}) \approx \frac{m_{0}^{2}(T)}{k_{B} T} V+\frac{D(d, \sigma ; \omega) d a^{d}}{(2 \pi)^{\sigma} \hat{J}(0) R_{0}^{\sigma}} L_{0}^{\sigma},
$$

where subsequent terms are $O\left(1, L_{0}^{2 \sigma-d}\right)$. These expressions again agree precisely with the results of the general scaling theory [16] in the limit $n \rightarrow \infty$ (although factors like $n /(n+2)$ regrettably remain unchecked). It should be remarked, however, that the lack of temperature dependence in the second term in (3.39) is an accident of the spherical model: more generally [16] there should appear a factor $m_{0}^{2}(T) / \Upsilon_{0}(T)$ which, for more realistic models, varies significantly with $T$ [19].

This completes our analysis of the block limit. We consider now cylinder shapes with one very long or even infinite dimension.

\section{Cylinder Shapes}

To discuss a cylinder, with periodic boundary conditions, in which one dimension, say $L_{1}$, can become indefinitely large or even infinite relative to the remaining dimensions, some supplementary notation is helpful. With

$$
N_{1} \equiv N_{\|}, \quad L_{1} \equiv L_{\|}, \quad \theta_{1} \equiv \theta_{\|}, \quad p_{1} \equiv p_{\|}, \quad \text { etc. }
$$

we write

$$
\mathbf{N}=\left(N_{\|}, \mathbf{N}_{\perp}\right), \quad \mathbf{L}=\left(L_{\|}, \mathbf{L}_{\perp}\right), \quad \boldsymbol{\theta}=\left(\theta_{\|}, \boldsymbol{\theta}_{\perp}\right), \quad \mathbf{p}=\left(p_{\|}, \mathbf{p}_{\perp}\right), \quad \text { etc. },
$$

and define the cross-sectional area

$$
A \equiv \prod_{k=2}^{d} L_{k} \equiv L_{\perp}^{d-1} \equiv\left(N_{\perp} a\right)^{d-1} .
$$

Since we wish to study the rounding of a first-order transition in a system of finite cross-section even in the limit $L_{\|}=\infty$ we must take $\sigma \geqq 1$, to exclude a transition in the latter case, but $\sigma<d$, to have a transition in the full bulk limit $\left(L_{\|}, A \rightarrow \infty\right)$. To avoid unilluminating mathematical complications, we will neglect the borderline case $\sigma=1$ and restrict attention here to range

$$
1<\sigma<d .
$$

To proceed we need an exact expression for the equation of state which parallels (3.1) but which suitably separates the longitudinal, $\|$, terms from the transverse, $\perp$, terms in such a way that the infinite cylinder limit $L_{\|} / L_{\perp} \rightarrow \infty$ may be taken. 
Accordingly, using (3.2) and (3.3) in (3.1), we remove from the sums on $\mathbf{p}$ those terms with $\mathbf{q}_{\perp}=\mathbf{p}_{\perp}=0$. Then, writing

$$
\Omega\left(\theta_{\|}, 0\right) \equiv \Omega_{\|}\left(\theta_{\|}\right) \approx\left(2 \pi \rho_{1} / N_{\|}\right)^{\sigma}\left|p_{\|}\right|^{\sigma}, \quad\left(\theta_{\|} \rightarrow 0\right),
$$

and regrouping yields

$$
2 K\left(m_{0}^{2}-m^{2}\right)=\frac{1}{N \phi}+\frac{1}{N_{\perp}^{d-1}} U_{0}^{\|}\left(\phi ; N_{\|}\right)-e_{0}^{\perp}(\mathbf{N})-\phi U_{1}^{\perp}(\phi ; \mathbf{N}),
$$

in which

$$
\begin{aligned}
U_{0}^{\|}\left(\phi ; N_{\|}\right) & =\frac{1}{N_{\|}} \sum_{p_{1} \neq 0} \frac{1}{\phi+\Omega_{\|}\left(\theta_{1}\right)}, \\
e_{0}^{\perp}(\mathbf{N}) & =\int_{\Theta} \frac{1}{\Omega(\boldsymbol{\theta})}-\frac{1}{N_{\mathbf{p}_{\perp} \neq 0}} \sum_{\frac{1}{\Omega(\boldsymbol{\theta})}}, \\
U_{1}^{\perp}(\phi, \mathbf{N}) & =\frac{1}{N_{\mathbf{p}_{\perp} \neq 0}} \sum_{\frac{1}{[\phi+\Omega(\boldsymbol{\theta})] \Omega(\boldsymbol{\theta})}} \frac{1}{}
\end{aligned}
$$

Apart from the restriction $\mathbf{p}_{\perp} \neq 0$ in place of $\mathbf{p} \neq 0$, the last two definitions precisely parallel (3.2) and (3.3).

For large $N_{\|}$and small $\phi$ we may use (4.5) in (4.7) to obtain the asymptotic result

$$
U_{0}^{\|}\left(\phi ; N_{\|}\right) \approx W(w) / \rho_{1} \phi^{(\sigma-1) / \sigma},
$$

where the scaled argument, to be compared with (3.18) and (3.28), is

$$
w=\frac{\phi N_{\|}^{\sigma}}{\left(2 \pi \rho_{1}\right)^{\sigma}}=\frac{d a^{d} k_{B} T h L_{1}^{\sigma}}{(2 \pi)^{\sigma} \hat{J}(0) m R_{1}^{\sigma}},
$$

while the scaling function is

$$
W(w)=\frac{1}{\pi} \sum_{p=1}^{\infty} \frac{w^{-1 / \sigma}}{1+\left(p^{\sigma} / w\right)}
$$

which satisfies

$$
\begin{aligned}
W(w) & \approx \pi^{-1} \zeta(\sigma) w^{(\sigma-1) / \sigma}, \quad \text { as } \quad w \rightarrow 0+, \\
W(\infty) & =1 / \sigma \sin (\pi / \sigma) .
\end{aligned}
$$

Consider next the truncation error, $e_{0}^{\perp}\left(N_{\|}, \mathbf{N}_{\perp}\right)$, in the limit $N_{\|} \rightarrow \infty$. The sum on $p_{1}\left(\equiv p_{\|}\right)$then becomes an integral and with $d^{\prime}=d-1$ one obtains

$$
e_{0}^{\perp}(\infty, \mathbf{N})=\left(\int \frac{d \boldsymbol{\theta}_{\perp}}{(2 \pi)^{d^{\prime}}}-\frac{1}{N_{\perp}^{d^{\prime}}} \sum_{\mathbf{p}_{\perp} \neq 0}\right) g_{\perp}\left(\boldsymbol{\theta}_{\perp}\right)
$$

where one finds that the operand behaves as

$$
g_{\perp}\left(\boldsymbol{\theta}_{\perp}\right) \approx\left[\Gamma\left(\frac{1}{2} \sigma^{\prime}\right) / 2 \sqrt{\pi} \Gamma\left(\frac{1}{2} \sigma\right) \rho_{1}\right]\left(\sum_{k^{\prime}=1}^{d^{\prime}} \rho_{k^{\prime}+1}^{2} \theta_{k^{\prime}+1}^{2}\right)^{-\sigma^{\prime} / 2},
$$

when $\left|\boldsymbol{\theta}_{\perp}\right| \rightarrow 0$, with $\sigma^{\prime}=\sigma-1$. An appeal to Appendix A then establishes the 
asymptotic form

$$
e_{0}^{\perp}\left(\infty, \mathbf{N}_{\perp}\right) \approx \frac{\Gamma\left(\frac{1}{2} \sigma-\frac{1}{2}\right) D\left(d-1, \sigma-1, \boldsymbol{\omega}_{\perp}\right)}{2 \sqrt{\pi} \Gamma\left(\frac{1}{2} \sigma\right)\left(2 \pi \rho_{\perp}\right)^{\sigma-1} \rho_{1} N_{\perp}^{d-\sigma}}
$$

where $\rho_{\perp}^{d-1}=\prod_{k=2}^{d} \rho_{k}$ and the cross-sectional shape enters through $\omega_{\perp}$, defined in analogy to (2.21). Evidently, as in the block case, the truncation error is essentially proportional to $1 / L_{\min }^{d-\sigma}$.

One may further consider the difference

$$
\Delta e_{0}^{\perp}(\mathbf{N}) \equiv e_{0}^{\perp}(\mathbf{N})-e_{0}^{\perp}\left(\infty, \mathbf{N}_{\perp}\right)=\left(\int \frac{d \theta_{1}}{2 \pi}-\frac{1}{N_{\|}} \sum_{p_{1}}\right) \frac{1}{N_{\perp}^{d-1}} \sum_{\mathbf{p}_{\perp} \neq 0} \frac{1}{\Omega\left(\theta_{1}, \boldsymbol{\theta}_{\perp}\right)},
$$

which vanishes as $N_{\|} \rightarrow \infty$. We have not analyzed this in details but one may anticipate that it decays like $1 / N_{\|}^{d-\sigma}$ times a bounded factor depending on the shape through $\omega$ [see (2.21)].

The last contribution, $U_{1}^{\perp}(\phi, \mathbf{N})$, can be analyzed as in Sect. 3 in parallel to $U_{1}(\phi, \mathbf{N})$. For our present purposes it suffices to approximate the sum by an integral to conclude

$$
\phi U_{1}^{\perp}(\phi, N)=O\left(\phi^{\delta}\right), \quad \delta=\min [1,(d / \sigma)-1], \quad \text { as } \phi \rightarrow 0 .
$$

Asymptotic Equation of State. On collecting the asymptotic results the equation of state can be written

$$
m_{0}^{2}-m^{2}=\frac{m}{V h}+\left(\frac{d k_{B} T}{\widehat{J}(0)}\right)^{1 / \sigma} \frac{a^{(d-\sigma) / \sigma}}{\rho_{1} A}\left(\frac{m}{h}\right)^{(\sigma-1) / \sigma} W(w)+O\left[(h / m)^{\delta}, L_{\perp}^{-d+\sigma}, L_{\|}^{-d+\sigma}\right],
$$

where $w\left(h / m, T ; L_{1}\right)$ is given by (4.11). This may be compared with the block result (3.26); the remainder terms here evidently describe the background terms of order $(h / m)^{i}(i=1,2, \ldots)$ and the spin-wave corrections which are controlled in relative magnitude by the parameter $u \propto L_{0}^{-d+\sigma}$ defined in (3.29). We will not discuss these higher order effects explicitly for cylinders.

To elucidate the scaling features of (4.20), consider first the zero-field susceptibility, $\chi_{0} \equiv \lim _{h \rightarrow 0}(m / h)$, of an infinite cylinder for $T<T_{c}$. From (4.20) one finds

$$
\chi_{0}^{\infty} \equiv \chi\left(0, T ; \infty, \mathbf{L}_{\perp}\right) \approx\left(\frac{\hat{J}(0)}{d a^{d-\sigma}}\right)^{1 /(\sigma-1)}\left[\sigma \sin \left(\frac{\pi}{\sigma}\right) \frac{\rho_{1} m_{0}^{2} A}{k_{B} T}\right]^{\sigma /(\sigma-1)},
$$

which, for short-range interactions $(\sigma=2)$ yields a divergence as $A^{2}$ (in agreement with [16-18]).

Longitudinal Correlation Length. Now, guided by the heuristic arguments [16-18] we would like to introduce a new scaled field variable

$$
y_{A}=h m_{0}(T) A \xi_{\|}(T ; A),
$$

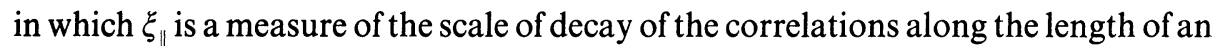
infinite cylinder of finite cross-section. In the case of short-range interactions this decay is exponential and is controlled by the location, at $q_{1}= \pm i / \xi_{\|}$, of two poles in 
the zero-field propagator or susceptibility $\hat{\chi}_{0}^{\infty}\left(q_{1}, \mathbf{q}_{\perp}=0\right)$, these being the closest singularities to the real $q_{1}$ axis. The propagator is always proportional to $1 /\left[\phi\left(0, T ; \infty, \mathbf{L}_{\perp}\right)+\Omega\left(q_{1} a, 0\right)\right]$ but, for general $\sigma$, is singular at $q_{1}=0$, resulting in a scale-free, power law component in the decay. However, poles which yield the longest spatial scale are still present and we use them to define $\xi_{\|}$by taking

$$
\begin{aligned}
\xi_{\|} & =R_{1} /\left[\phi\left(0, T ; \infty, \mathbf{L}_{\|}\right)\right]^{1 / \sigma}=\rho_{1}\left[\hat{J}(0) \chi_{0}^{\infty} / d a^{d-\sigma}\right]^{1 / \sigma}, \\
& \approx\left(\frac{\sigma \sin (\pi / \sigma) \rho_{1}^{\sigma} \hat{J}(0) m_{0}^{2}}{d a^{d-\sigma} k_{B} T} A\right)^{1 /(\sigma-1)} \equiv X_{\|}(T) A^{1 /(\sigma-1)} .
\end{aligned}
$$

For nearest-neighbor interactions of strength $J_{0} \equiv \widehat{J}(0) / 2 d$ we have $\sigma=2$ and $\rho_{1}=1$, and so obtain

$$
\xi_{\|}(T ; A) \approx 4 J_{0} m_{0}^{2}(T) A / a^{d-2} k_{B} T .
$$

According to the phenomenological theory $[16,17]$ this correlation length should be related via (1.4) to the helicity modulus, $Y(T)$ [19]. This relation is, in fact, precisely verified since for the spherical model one has [6]

$$
\Upsilon(T) \equiv \lim _{n \rightarrow \infty} \Upsilon_{(n)} /(n-1)=2 J_{0} m_{0}^{2}(T) / a^{d-2} .
$$

More generally, for spatially anisotropic interactions of finite range one may, as mentioned in Sect. 3, introduce a helicity modulus tensor with diagonal components $\Upsilon_{k}(T)(k=1, \ldots, d)$. By using (4.23) for other orientations of the cylinder axis, one sees for any short-range spherical model that this should be given by $\Upsilon_{k}(T)=$ $\left[\hat{J}(0) R_{k}^{2} / d a^{d}\right] m_{0}^{2}(T)$ in agreement with (3.31).

Scaling Forms. With the aid of the scaled variable $y_{A}$ we may write the equation of state for an infinite cylinder asymptotically as

$$
m\left(H, T ; \infty, \mathbf{L}_{\perp}\right) \approx m_{0}(T) Y_{\infty}\left(y_{A}\right)
$$

The scaling function, $Y_{\infty}$, follows from (4.20) in which $w \rightarrow \infty$ by (4.11): it is the solution of the equation

$$
Y_{\infty} /\left(1-Y_{\infty}^{2}\right)^{\sigma /(\sigma-1)}=\sigma \sin (\pi / \sigma) y_{A} .
$$

For short range forces $(\sigma=2)$, this expression confirms the result obtained in [16] for the limit $n \rightarrow \infty$.

Finally, we expect [16] the crossover from the cylinder limit, $L_{1} / L_{\perp} \rightarrow \infty$, to the block limit, $L_{1} / L_{\perp}$ and $L_{k} / L_{0}$ bounded, to be described by

$$
m(H, T ; \mathbf{L}) \approx m_{0} Y\left(y_{V}, y_{A}\right),
$$

where, as before, $y_{V}=m_{0} h V$. To derive an equation for the two-variable scaling function, $Y$, note that we can rewrite (4.11) in scaled form as

$$
w=(2 \pi)^{-\sigma} \sigma \sin (\pi / \sigma)\left(y_{V} / y_{A}\right)^{\sigma}\left[y_{A} / Y\left(y_{V}, y_{A}\right)\right] .
$$

Then (4.20) yields

$$
1-Y^{2}=\left(Y / y_{V}\right)+[\sigma \sin (\pi / \sigma)]^{1 / \sigma}\left(Y / y_{A}\right)^{(\sigma-1) / \sigma} W(w)
$$


in which $w$ is to be given by (4.29).

To check that this reduces correctly for long cylinders note that

$$
y_{V}=y_{A}\left[L_{\|} / L_{\perp}^{\tau} X_{\|}(T)\right] \quad \text { with } \tau=(d-1) /(\sigma-1)
$$

where $X_{\|}$is defined by (4.23), and we can write

$$
w=y_{A}\left(L_{\|} / L_{\perp}^{\tau}\right)^{\sigma}\left[\sigma \sin (\pi / \sigma) /\left(2 \pi X_{\|}\right)^{\sigma} Y\right] .
$$

Thus $y_{V}$ and $w$ diverge at fixed $y_{A}$ when $L_{\|} / L_{\perp}^{\tau} \rightarrow \infty$ and (4.27) is then recaptured. To understand the other limit note that one also has

$$
w=y_{V} u(T ; \mathbf{L}) /\left(2 \pi \sqrt{\omega_{1}}\right)^{\sigma} Y,
$$

where $\omega_{1}=\left(\rho_{1} / \rho_{0} l_{1}\right)^{2}$, as before, while $u \sim 1 / L_{0}^{d-\sigma}$ is the correction parameter of (3.29). Hence, in the block limit at fixed $y_{V}$, one has $w \rightarrow 0$ and, on appealing to (4.13), one finds that the last, i.e. the cylinder term in (4.30) vanishes as

$$
2 \zeta(\sigma)\left(\rho_{0} l_{1} / 2 \pi \rho_{1}\right)^{\sigma} u(T ; \mathbf{L})[1+o(w)] .
$$

On comparing with the full block scaling form (4.20) one sees that cylinder term can be regarded merely as part of the spin wave corrections to scaling: clearly, once $L_{\|} / L_{\perp}^{\tau}$ is sufficiently small that $L_{\|} / L_{0}$ is of order unity the terms originally neglected in (4.20) become of comparable importance to the cylinder term. Conversely, the cylinder term is only significant when $L_{\|} / L_{0}$ becomes large and then appears as a "dangerous correction to scaling" [21].

This completes our analysis of finite-size effects at first-order transitions in general ferromagnetic spherical models. The asymptotic behavior in block and cylinder limits is described by universal scaling functions, and the exact expressions confirm in detail the general but nonrigorous theory [16-18] for $n$-component spin systems when the limit $n \rightarrow \infty$ is taken.

\section{Appendix: Approach of a Sum to a Multivariate Singular Integral}

Let $g(\boldsymbol{\theta}) \equiv g\left(\theta_{1}, \ldots, \theta_{d}\right)$ be a function periodic in each argument, $\theta_{j}$, with period $2 \pi$, which is real and analytic in $\boldsymbol{\theta}$ except at $\boldsymbol{\theta}=0$, where it diverges as

$$
g(\boldsymbol{\theta})=\left[\sum_{j=1}^{d} \rho_{j}^{2} \theta_{j}^{2}\right]^{-\sigma / 2}\left[1+O\left(|\boldsymbol{\theta}|^{\delta}\right)\right]
$$

with $\sigma, \delta>0$ and

$$
\rho_{j}>0(\text { all } j) \quad \text { and } \quad \rho_{0}^{d} \equiv \prod_{j=1}^{d} \rho_{j} .
$$

In addition we assume $\sigma<d$, so that the integral

$$
I \equiv I(d, \sigma ; \boldsymbol{\rho})=\int_{\boldsymbol{\theta}} g(\boldsymbol{\theta}) \equiv \int_{-\pi}^{\pi} \frac{d \theta_{1}}{2 \pi} \cdots \int_{-\pi}^{\pi} \frac{d \theta_{d}}{2 \pi} g(\boldsymbol{\theta})
$$

over the basic zone, $\Theta$, is well-defined. To construct an approximating sum we let 
the integer vector $\mathbf{N} \equiv\left(N_{1}, \ldots, N_{d}\right)$ with

$$
N \equiv N_{0}^{d} \equiv \prod_{j=1}^{d} N_{j} \quad \text { and } \quad N_{j}=l_{j} N_{0} \quad(j=1, \ldots, d),
$$

become infinite in such a way that the ratios $l_{j}$ approach bounded, nonzero limits. Then we may discretize the variable $\boldsymbol{\theta}$ via

$$
\boldsymbol{\theta}_{\mathbf{p}} \equiv\left[\left(\boldsymbol{\theta}_{\mathbf{p}}\right)_{j}=2 \pi p_{j} / N_{j}\right],
$$

where $\mathbf{p}$ is a vector with (positive or negative) integral components, $p_{j}$, identified modulo $N_{j}$. The identification $g_{\mathbf{p}} \equiv g\left(\boldsymbol{\theta}_{\mathbf{p}}\right)$ fails for $\mathbf{p}=0\left(p_{j}=0\right.$, all $\left.j\right)$, so it is natural to define an approximating sum and difference via

$$
\Delta_{\mathrm{N}} \equiv \Delta_{\mathrm{N}}(d, \sigma ; \boldsymbol{\rho})=I-S_{\mathrm{N}}=I-\frac{1}{N} \sum_{\mathrm{p} \neq 0} g_{\mathrm{p}} .
$$

Note that the sum contains $N-1$ elements.

The aim of this appendix is to demonstrate that as $N_{0} \rightarrow \infty$ the difference between integral and sum vanishes according to

$$
\Delta_{\mathrm{N}} \approx D(d, \sigma ; \boldsymbol{\omega}) /\left(2 \pi \rho_{0}\right)^{\sigma} N_{0}^{d-\sigma}
$$

where $\boldsymbol{\omega}=\left(\omega_{j}\right)$ and

$$
\omega_{j}=\left(\rho_{j} / \rho_{0} l_{j}\right)^{2},
$$

and to show how expressions for the coefficient $D$ may be obtained. The terms neglected in (A7) are of higher order in $1 / N_{0}$, but will not be bounded explicitly. For ease of exposition we discuss first the simplest case $d<\sigma+2$. Next we consider the range $\sigma+2 \leqq d<\sigma+4$; how to handle the higher order cases, $\sigma+4 \leqq d<\sigma+6$, etc., will then be apparent.

To make progress we decompose the integral, $I$, into a sum of terms. To that end let $\gamma(\mathbf{p})$ denote the rectangular cell in $\theta$-space centered on $\boldsymbol{\theta}_{\mathbf{p}}$, and defined explicitly by $\theta_{j}=2 \pi\left(p_{j}+v_{j}\right) / N_{j}$ with $-\frac{1}{2} \leqq v \leqq \frac{1}{2}$. We then have

$$
I=\sum_{\mathbf{p}} I_{\mathbf{p}, \mathbf{N}} \quad \text { with } \quad I_{\mathbf{p}, \mathbf{N}}=\int_{\gamma(\mathbf{p})} g(\boldsymbol{\theta}),
$$

where the integral is defined by analogy with (A3). The origin term, $I_{0, \mathrm{~N}}$, may be evaluated easily when $N_{0} \rightarrow \infty$, using (A1) which yields

$$
A_{\mathrm{N}} \equiv I_{0, \mathrm{~N}} \approx D_{0}(\omega) /\left(2 \pi \rho_{0}\right)^{\sigma} N_{0}^{d-\sigma},
$$

in which the numerical coefficient can be written

$$
D_{0}(\boldsymbol{\omega})=\left\langle\left[\sum_{j=1}^{d} \omega_{j} v_{j}^{2}\right]^{-\sigma / 2}\right\rangle,
$$

where, for any function, $f(\mathbf{v})$, the cell average is defined by

$$
\langle f(\mathbf{v})\rangle=\int_{-1 / 2}^{1 / 2} d v_{1} \ldots \int_{-1 / 2}^{1 / 2} d v_{d} f\left(v_{1}, \ldots, v_{d}\right) .
$$


Substitution of (A8) in (A6) and rearrangement yields

$$
\Delta_{\mathrm{N}}=A_{\mathrm{N}}+B_{\mathrm{N}}=A_{\mathrm{N}}+\frac{1}{N} \sum_{\mathrm{p} \neq 0} b_{\mathrm{p}}
$$

where

$$
b_{\mathbf{p}}=\left\langle b_{\mathbf{p}}(\mathbf{v})\right\rangle \quad \text { with } \quad b_{\mathrm{p}}(\mathbf{v})=g_{\mathrm{p}}(\mathbf{v})-g_{\mathrm{p}}(0),
$$

in which, here and below, we take

$$
g_{\mathbf{p}}(\mathbf{v})=g\left(\frac{2 \pi}{N_{1}}\left(p_{1}+v_{1}\right), \ldots, \frac{2 \pi}{N_{d}}\left(p_{d}+v_{d}\right)\right) .
$$

Now if, in place of (A1), the periodic function $g(\boldsymbol{\theta})$ were analytic at $\boldsymbol{\theta}=0$ the correction $\Delta_{\mathrm{N}}$ would decay exponentially fast with $N_{0}$ [6]. Conversely, any slower decay must be associated only with the behavior of $g(\boldsymbol{\theta})$ near the origin that is for $\left|\boldsymbol{\theta}_{\mathbf{p}}\right| \ll 1$ or $|\mathbf{p}| \ll N_{0}$. In this region we can use (A1) and thus have

$$
b_{\mathbf{p}}(\mathbf{v}) \approx\left(N_{0} / 2 \pi \rho_{0}\right)^{\sigma} c_{\mathbf{p}}(\mathbf{v} ; \boldsymbol{\omega})
$$

where

$$
c_{\mathrm{p}}(\mathbf{v} ; \boldsymbol{\omega})=[P(\mathbf{v})]^{-\sigma}-P_{0}^{-\sigma},
$$

in which, for brevity, we have written

$$
P^{2}(\mathbf{v})=\sum_{j=1}^{d} \omega_{j}\left(p_{j}+v_{j}\right)^{2} \equiv P_{0}^{2}+2 \sum_{j=1}^{d} \omega_{j}\left(p_{j}+\frac{1}{2} v_{j}\right) v_{j}
$$

with $P_{0} \equiv P(\mathbf{v}=0)$.

To investigate the coefficients $b_{\mathrm{p}}$, let us expand $b_{\mathrm{p}}(\mathbf{w})$ in a Taylor series about $\mathbf{w}=0$, and average using (A12). This yields

$$
\bar{b}_{\mathbf{p}}=\frac{1}{24} \sum_{j=1}^{d}\left(\frac{\partial^{2} b}{\partial v_{j}^{2}}\right)_{\mathbf{p}}+\frac{1}{1152} \sum_{i, j=1}^{d}\left(1-\frac{2}{5} \delta_{i j}\right)\left(\frac{\partial^{4} b}{\partial v_{1}^{2} \partial v_{j}^{2}}\right)_{\mathbf{p}}+\cdots,
$$

where the subscript $\mathbf{p}$ on the derivatives indicates evaluation at $\mathbf{v}=0$. For $|\mathbf{p}| \ll N_{0}$ we thus have, in particular,

$$
\left(\partial^{2} b / \partial v_{j}^{2}\right)_{\mathbf{p}} \approx\left(N_{0} / 2 \pi \rho_{0}\right)^{\sigma}\left(\partial^{2} c_{\mathbf{p}} / \partial v_{i}^{2}\right)_{v=0},
$$

where

$$
\left(\frac{\partial^{2} c}{\partial v_{j}^{2}}\right)_{\mathbf{p}}(\mathbf{v})=-\frac{\sigma \omega_{j}}{[P(\mathbf{v})]^{\sigma+2}}\left[1-(\sigma+2) \omega_{j} \frac{\left(p_{j}+v_{j}\right)^{2}}{P^{2}(\mathbf{v})}\right] .
$$

On noting that $\omega_{j}\left(p_{j}+v_{j}\right)^{2} / P^{2}(\mathbf{v})$ is bounded by unity for all $\mathbf{p}$, we see that the secondorder $b$ derivative is of order $1 / P_{0}^{\sigma+2}$ when $|\mathbf{p}| \rightarrow \infty$, while the subsequent terms in (A19) are of order $1 / P_{0}^{\sigma+2 k}$ with $k=2,3, \ldots$.

We may now consider the asymptotic replacement of the coefficient $\bar{b}_{\mathrm{p}}$ in (A13) implied by (A16). The convergence of the sum on $\mathbf{p}$ in (A13) as $N_{0} \rightarrow \infty$ is then like that of $\int_{0}^{N_{0} / 2} \bar{c}_{p} p^{d-1} d p$. Thus, accepting the estimate $\bar{c}_{\mathrm{p}}=\left\langle c_{\mathrm{p}}\right\rangle=O\left(1 / P_{0}^{\sigma+2}\right)=$ 
$O\left(1 /|\mathbf{p}|^{\sigma+2}\right)$, which follows from (A19)-(A21), we see that the upper limits on $\mathbf{p}$ in the sum may be neglected asymptotically provided $\sigma+2>d$ : this is just the first case anticipated above. Collecting up terms and recalling (A10) finally confirms the claim (A7) and yields the coefficient

$$
D=D_{0}(\boldsymbol{\omega})+\sum_{\mathbf{p} \neq 0}\left\langle c_{\mathbf{p}}(\mathbf{v}: \boldsymbol{\omega})\right\rangle,
$$

where (A11), (A12), (A17) and (A18) provide the necessary definitions, while the sum runs over all nonzero $\mathbf{p}$ and converges absolutely when $\sigma+2>d$.

To deal with the case $\sigma+2 \leqq d$, it is evidently necessary to obtain a more rapidly convergent expression for $D$ than (A22). To this end we endeavor to find a modification of the coefficient, $\bar{b}_{\mathbf{p}}$, in which the leading large $|\mathbf{p}|$ behavior, fixed by the second derivative term in (A19), is cancelled out. Accordingly consider the region $\Theta^{-}=\Theta \backslash \gamma(\mathbf{0})$, i.e., the whole $\theta$ zone but with the origin cell, $\gamma(\mathbf{0})$, deleted, and the corresponding integrals

$$
Q_{\mathbf{N}}^{j}=\int_{\Theta^{-}}\left(\frac{\partial^{2} g}{\partial \theta_{1}^{2}}\right)=\sum_{\mathbf{p} \neq 0} Q_{\mathbf{p}, \mathbf{N}}^{j},
$$

in which $Q_{\mathbf{p}, \mathbf{N}}^{j}$ is the same form of integral but restricted to the cell $\gamma(\mathbf{p})$. Now, except for the deleted cell, $\gamma(\mathbf{0})$, the integral over $\Theta^{-}$represents an integral of a derivative of an analytic function: consequently, its value is entirely determined by the function on the boundary of $\gamma(\mathbf{0})$. (Of course, this is just an example of the divergence theorem.) Explicitly we find

$$
Q_{\mathrm{N}}^{j}=-\int_{\partial_{j} \gamma(\mathbf{0})} \frac{1}{2 \pi} \operatorname{sgn}\left(\theta_{j}\right)\left(\frac{\partial g}{\partial \theta_{j}}\right),
$$

where the $(d-1)$-fold integral runs over the two faces of $\gamma(\mathbf{0})$ normal to the $\theta_{j}$ axis. By using (A15) this may be rewritten

$$
Q_{\mathrm{N}}^{j}=-\frac{2}{N}\left(\frac{N_{j}}{2 \pi}\right)^{2}\left\langle\operatorname{sgn}\left(v_{j}\right)\left(\frac{\partial g_{0}}{\partial v_{j}}\right)_{v_{j}= \pm \frac{1}{2}}\right\rangle,
$$

where the + or - is determined by the sign of $v_{j}$. Finally, for $|\mathbf{p}| \ll N_{0}$ we have, as before, $\left|\theta_{p}\right| \ll 1$ and can use (A1) to estimate

$$
Q_{\mathrm{N}}^{j} \approx D_{j}(\omega)\left(N_{j} / 2 \pi\right)^{2} /\left(2 \pi \rho_{0}\right)^{\sigma} N_{0}^{d-\sigma}
$$

where

$$
D_{j}(\boldsymbol{\omega})=\sigma \omega_{j}\left\langle\left[\frac{1}{4} \omega_{j}+\sum_{i \neq j}^{d} \omega_{i} v_{i}^{2}\right]^{-(\sigma+2) / 2}\right\rangle .
$$

On the other hand, we may analyze the individual integrals in the sum in (A23). On rewriting in terms of $b_{\mathbf{p}}(\mathbf{v})$ we have

$$
\begin{aligned}
Q_{\mathrm{p}, \mathrm{N}}^{j} & =N^{-1}\left(N_{j} / 2 \pi\right)^{2}\left\langle\partial^{2} b_{\mathrm{p}}(\mathbf{v}) / \partial v_{j}^{2}\right\rangle \\
& =\frac{1}{N}\left(\frac{N_{j}}{2 \pi}\right)^{2}\left[\left(\frac{\partial^{2} b}{\partial v_{j}^{2}}\right)_{\mathrm{p}}+\frac{1}{24} \sum_{k=1}^{d}\left(\frac{\partial^{4} b}{\partial v_{j}^{2} \partial v_{k}^{2}}\right)_{\mathrm{p}}+\cdots\right],
\end{aligned}
$$


where the second line follows by the reasoning that lead to (A19) and employs the same notation. By the same token, the leading correction, arising from the fourthorder derivatives, decreases as $1 / P_{0}^{\sigma+4}$ (in the region $|\mathbf{p}| \ll N_{0}$ ). Consequently, by comparing (A19) and (A28) we see that the modified coefficients

$$
\bar{b}_{\mathbf{p}}^{(2)}=\bar{b}_{\mathbf{p}}-\frac{N}{24} \sum_{j=1}^{d} \frac{4 \pi^{2}}{N_{j}^{2}} Q_{\mathbf{p}, \mathbf{N}}^{j} \approx\left(\frac{N_{0}}{2 \pi \rho_{0}}\right)^{\sigma}\left\langle c_{\mathbf{p}}^{(2)}\right\rangle
$$

decay as $1 / P_{0}^{\sigma+4}=O\left(|\mathbf{p}|^{-\sigma-4}\right)$, when $|\mathbf{p}| \gg 1$ (with $\left.|\mathbf{p}| \ll N_{0}\right)$; likewise the corresponding modified functions

$$
c_{\mathbf{p}}^{(2)}(\mathbf{v} ; \boldsymbol{\omega})=\left[1-\frac{1}{24} \nabla_{v}^{2}\right] c_{\mathbf{p}}(\mathbf{v} ; \boldsymbol{\omega})=c_{\mathrm{p}}-\frac{1}{24} \sum_{j=1}^{d}\left(\partial^{2} c_{\mathbf{p}} / \partial v_{j}^{2}\right),
$$

yield, on averaging over $\mathbf{v}$, coefficients decreasing as $|\mathbf{p}|^{-\sigma-4}$. It follows that one may replace $\left\langle b_{\mathbf{p}}^{(2)}\right\rangle$ in a sum over $\mathbf{p}(\neq 0)$ by $\left\langle c_{\mathbf{p}}^{(2)}\right\rangle$, and extend the sum to infinity provided only that $\sigma+4$ exceeds $d$, a weaker condition than previously.

Consequently, on adding to and subtracting from $\Delta_{\mathrm{N}}$ the combination of integrals $Q_{\mathrm{p}, \mathrm{N}}^{j}$ corresponding to (A29), we rederive (A7), but with the amplitude given by

$$
D=D_{0}(\boldsymbol{\omega})+\frac{1}{24} \sum_{j=1}^{d} D_{j}(\boldsymbol{\omega})+\sum_{\mathbf{p} \neq 0}\left\langle c_{\mathbf{p}}^{(2)}(\mathbf{v} ; \boldsymbol{\omega})\right\rangle .
$$

The necessary definitions are (A11), (A12), and (A30), which entails (A17), (A18), and (A21). The sum runs over all nonzero integer vectors, $\mathbf{p}$, and converges absolutely when $d<\sigma+4$. Of course, this expression yields the same value as (A22) when the latter converges (as it does for $d<\sigma+2$ ).

How to extend the treatment to $d \geqq \sigma+4$ should now be clear. The procedure is to define, in analogy to (A30), $c_{\mathbf{p}}^{(4)}, c_{\mathrm{p}}^{(6)}, \ldots$ by subtracting off fourth order derivatives, sixth order derivatives, and so on. The correct linear combinations follow by using (A19), including the further terms, together with the corresponding terms arising in (A28) and from subsequent higher order subtractions. The combinatorics involved in the general term seem formidable but it is clear that explicit expressions valid for $d<\sigma+2 k$ for $k=3,4, \ldots$ can be obtained by brute force. The leading behavior of $\Delta_{N}$ is always of the form (A7). However, higher order corrections, which we have not discussed, arise in particular from the $O\left(|\theta|^{\delta}\right)$ terms in (A1) which clearly enter into the determination of the degree of approximation of $b_{\mathbf{p}}(\mathbf{v})$ by $c_{\mathbf{p}}(\mathbf{v})$ : see (A16) and (A17).

It is worth remarking that alternative tactics can be used which lead to expressions for $D$ which are simpler in that it is not necessary to do the integrations over $\nabla_{v}^{2} c_{\mathrm{p}}(\mathbf{v})$ implied by (A31). Instead of cancelling the second derivative term in $b_{\mathbf{p}}$ and $c_{\mathrm{p}}$ by adding and subtracting an integral over a continuous second derivative, one can use a sum over a discrete, lattice second derivative, say $\left[g_{\mathbf{p}+\mathbf{j}}+g_{\mathbf{p}-\mathbf{j}}-2 g_{\mathbf{p}}\right]$ (where $\mathbf{j}$ is a unit vector along the $j^{t h}$ axis). The simpler answers however, require a few more steps to derive.

It should also be mentioned that an alternative strategy to deriving (A7) can be based on the Ewald summation technique $[13,22]$. This approach yields, indeed, expressions which are computationally superior since they typically converge very 
rapidly: see Refs. [13,22,23] and the references cited, for numerical calculations for several values of $\sigma$. However, from an analytical viewpoint, the expressions obtained are less satisfactory (containing an essentially arbitrary "range splitting' parameter). The derivation presented here is also advantageous from a scaling view-point, since the parameters $\rho_{0}$ and $N_{0}$ enter very naturally, and the dependence of the amplitude $D$ on the shape/anisotropy parameters is seen at an early stage.

Acknowledgements. The authors have enjoyed instructive discussions with J. Rudnick and J. Shapiro and wish to acknowledge partial support from the U.S. National Science Foundation through the Condensed Matter Theory Program ${ }^{1}$ and from the Science and Engineering Research Council of the United Kingdom. M.E.F. is grateful to the California Institute of Technology for hospitality during his visit as a Sherman Fairchild Distinguished Scholar, and to the Department of Theoretical Physics at the University of Oxford, where this work was completed, for their hospitality. V.P. acknowledges the award of a Bantrell Fellowship by the California Institute of Technology.

1 Grants Nos. DMR-81-17011 and DMR-84-12543

\section{References}

1. Kac, M.: Phys. Today 17, 40-42 (1964)

2. Berlin, T. H., Kac, M.: The spherical model of a ferromagnet. Phys. Rev. 86, 821-835 (1952)

3. Montroll, E. W.: Nuovo Cimento, Suppl. 6, 265-278 (1949)

4. Lewis, H. W., Wannier, G. H.: Spherical model of a ferromagnet. Phys. Rev. 88, 682-683 (1952)

5. Joyce, G. S.: In: Phase transitions and critical phenomena, Vol. II, Domb C., Green, M. S. (eds.) p. 375-492, London: Academic 1972

6. Barber, M. N., Fisher, M. E.: Critical phenomena in systems of finite thickness, I. The spherical model. Ann. Phys. 77, 1-78 (1973). Note that Eqs. (10.28) and (10.29) lack a factor of 2 and, for dimensional consistency, $a^{-d}$

7. Barber, M. N.: Critical behavior of a spherical model with a free surface. J. Stat. Phys. 10, 59-88 (1974)

8. Fisher, M. E.: In Critical phenomena. Proc. Enrico Fermi Int. School of Physics, Vol. 51, Green, M. S. (ed.) N.Y.: Academic 1971, p. 1-99

9. Fisher, M. E., Barber, M. N. Scaling theory for finite size effects in the critical region. Phys. Rev. Lett. 28, 1516-1519 (1972)

10. Barber, M. N.: In: Phase transitions and critical phenomena, Vol. VIII, Domb, C., Lebowitz, J. L. (eds.). N.Y.: Academic 1983, p. 145-266.

11. Brézin, E.: Ann. N.Y. Acad. Sci. 410, 339-349 (1983)

12. Luck, J. M.: Corrections to finite-size-scaling laws and convergence of transfer-matrix methods. Phys. Rev. B31, 3069-3083 (1985); Singh, S., Pathria, R. K.: Phys. Rev. B31, 4483-90 (1985)

13. Shapiro, J., Rudnick, J.: The fully finite spherical model, to be published, and private communication

14. Stanley, H. E.: Spherical model as the limit of infinite spin dimensionality, Phys. Rev. 176, 718-722 (1968)

15. Kac, M., Thompson, C. J.: Phys. Norv. 5, 163-168 (1971)

16. Fisher, M. E., Privman, V.: First order transitions breaking $O(n)$ symmetry: finite-size scaling. Phys. Rev. B32, 447-464 (1985)

17. Privman, V., Fisher, M. E.: J. Appl. Phys. 57, 3327-28 (1985)

18. Privman, V., Fisher, M. E.: Finite-size effects at first-order transitions, J. Stat. Phys. 33, 385-417 (1983). Note a missing factor $z^{-d}$ on the righthand side of Eq. (5.6)

19. Fisher, M. E., Barber, M. N., Jasnow, D.: Helicity modulus, superfluidity, and scaling in isotropic systems. Phys. Rev. A8, 1111-24 (1973)

20. Kurtze, D. A., Fisher, M. E.: The Yang-Lee edge singularity in spherical models. J. Stat. Phys. 19, 205-218 (1978) 
21. Fisher, M. E.: In: Critical phenomena. Lecture Notes in Physics, Vol. 186, pp. 1-139, Hahne, F. J. W. (ed.) Berlin, Heidelberg, New York: Springer (1983) Appendix D

22. Harris, F. E., Monkhorst, H. J.: Electronic-structure studies of solids. I. Fourier representation method for Madelung sums. Phys. Rev. B2, 4400-4405 (1970)

23. Chaba, A. N., Pathria, R. K.: Evaluation of a class of lattice sums in arbitrary dimensions. J. Math. Phys. 16, 1457-1460 (1975)

Communicated by A. Jaffe

Received May 13, 1985 